\title{
Evaluation of Hematological Parameters in Predicting Intensive Care Unit Admission in COVID-19 Patients
}

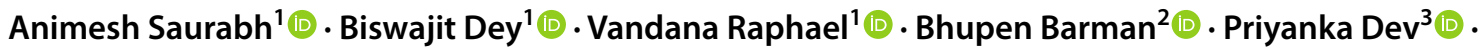

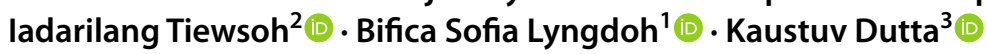

Accepted: 28 December 2021 / Published online: 17 January 2022

(C) The Author(s), under exclusive licence to Springer Nature Switzerland AG 2022

\begin{abstract}
Hematological parameters like total leukocyte count (TLC), neutrophil, lymphocyte, and absolute eosinophil counts (AEC), and neutrophil-to-lymphocyte ratio (NLR) are known to predict the severity of novel coronavirus disease 2019 (COVID19) patients. In the present study, we aimed to study the role of complete blood count parameters in triaging these patients requiring intensive care unit (ICU) admission. A retrospective study was done over a period of 2 months. Patients, who were $\geq 18$ years of age with COVID-19 confirmed on SARS-CoV-2 reverse transcription-polymerase chain reaction (RTPCR) and whose routine hematology counts were sent within $24 \mathrm{~h}$ of admission, were included in the study. Cut-off values of 47.5 years for age, $11.3 \times 10^{9} / \mathrm{L}$ for TLC, and 9.1 for NLR were predictive of disease severity among COVID-19 patients. Relative neutrophilia $\geq 70 \%(p<0.007)$, relative lymphopenia $\leq 20 \%(p<0.002)$, AEC $\leq 40 /$ cumm $(p<0.001)$, and NLR $\geq 9.1$ $(p<0.001)$ were significantly associated with ICU admission. Routine hematological parameters are cost-effective and fast predictive markers for severe COVID-19 patients, especially in resource-constrained health care settings to utilize limited ICU resources more effectively.
\end{abstract}

Keywords COVID-19 $\cdot$ Eosinophil $\cdot$ Lymphopenia $\cdot$ Neutrophils $\cdot$ Intensive care units

\section{Introduction}

Novel coronavirus disease 2019 (COVID-19) resembles severe acute respiratory syndrome coronavirus (SARS$\mathrm{CoV}$ ) [1]. Most patients are asymptomatic and those with

This article is part of the Topical Collection on Covid-19

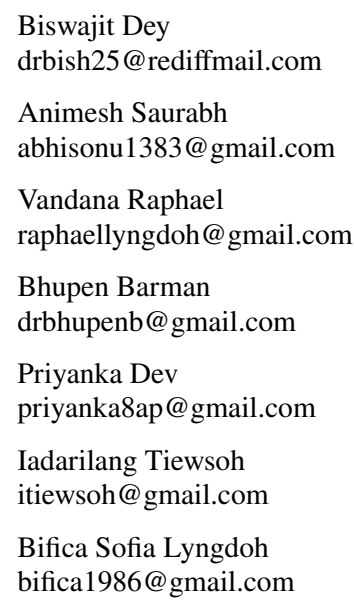

symptoms develop mild flu-like conditions to severe acute respiratory syndrome or death [1, 2]. Gastrointestinal symptoms are also reported in COVID-19 patients which include nausea and diarrhea [2]. Clinical signs of COVID-19 include fever, cough, expectorations, shortness of breath, and anosmia [2].

Kaustuv Dutta

kaustuv.dutta@gmail.com

1 Department of Pathology, North Eastern Indira Gandhi Regional Institute of Health and Medical Sciences, Shillong 793018, India

2 Department of Internal Medicine, North Eastern Indira Gandhi Regional Institute of Health and Medical Sciences, Shillong, India

3 Department of Anaesthesia and Critical Care, North Eastern Indira Gandhi Regional Institute of Health and Medical Sciences, Shillong, India 
Considering the infectivity and serious harm of COVID-19, it is of paramount importance to identify various circulating biomarkers, which can predict the severity of COVID-19 [1]. Complete blood counts (CBC) including total leukocyte count (TLC) and neutrophil-tolymphocyte ratio (NLR) are indicators of the systematic inflammatory response that are being widely investigated as predictors of severity of COVID-19 pneumonia [1]. Lymphocyte and eosinophil counts, which are indicators of inflammation, have also been widely used for predicting severity in COVID-19 patients [1,3]. Because of the large number of COVID-19 patients flooding the healthcare system, these routine markers are especially important. Therefore, a simple CBC which includes TLC, neutrophil, lymphocyte, and eosinophil counts, and NLR may be extremely useful in predicting the severity and triaging of these patients especially in developing countries with limited resources.

In light of this, the current study was conducted to investigate the role of CBC parameters like TLC, neutrophil, lymphocyte, and absolute eosinophil counts in triaging the patients requiring intensive care unit (ICU) admission.

\section{Methods}

\section{Study Design}

This is a retrospective study done over a period of 2 months from 1st April 2021 to 31st May 2021. All patients, who were $\geq 18$ years of age with COVID-19 confirmed on SARS-CoV-2 reverse transcription-polymerase chain reaction (RT-PCR) and whose routine hematology counts were sent within $24 \mathrm{~h}$ of admission were included in the study. Patients already on steroid treatment were excluded from the study.

\section{Laboratory Testing}

Blood samples were collected in vacutainers containing EDTA (ethylenediamine tetraacetic acid) for CBC. CBC of the patients were done on automated six parts Sysmex hematology analyzer. The following parameters were assessed: hemoglobin, platelets count, total leukocyte counts (TLC), relative neutrophil count, relative lymphocyte count, neutrophil-to-lymphocyte ratio (NLR), and absolute eosinophil counts (AEC).

Anemia was defined as hemoglobin value $<12$ gm\%. Thrombocytopenia was defined as platelets count $\leq 150 \times 10^{9} / \mathrm{L}$. Relative neutrophilia was defined as neutrophil $\geq 70 \%$, and relative lymphopenia was defined as lymphocytes $\leq 20 \%$. Eosinopenia was defined as AEC $\leq 40$ / cumm.

\section{Criteria for ICU Admission}

Patients having any one of these-respiratory rate $>30 / \mathrm{min}$, breathlessness, or $\mathrm{SpO} 2<90 \%$ on room air-were admitted to ICU $[4,5]$.

\section{Statistical Analysis}

We grouped the patients into ICU and non-ICU according to the criteria stated above. The optimal cut-off values of continuous NLR, age, and TLC were calculated by applying the receiver operating characteristic (ROC) curve analysis. All the variables were summarized as percentages and counts in each category. Their association with ICU and non-ICU admission was evaluated by the chi-square $(\chi 2)$ test and Fisher's exact test. A $p$-value $<0.05$ was recognized as statistically significant. All the statistical calculations were performed by Statistical Package for Social Science (SPSS) Software 64-bit version.

\section{Results}

A total of 105 patients who were positive for SARS-Cov2 on RT-PCR were included in the study. The age of the patients ranged from 18 to 82 years with a mean age of 48.8 years and median age of 50 years. There were 54 males and 51 female patients with a M:F ratio of 1.05 . Out of 105 patients admitted, 55 patients required ICU care.

The range and median values of the hematological parameters of the patients are tabulated in Table 1.

Age, TLC, and NLR were used to identify patients who required ICU admission. No single accepted reference value was found for these parameters. Therefore, we analyzed the optimal cut-off values of age, TLC, and NLR calculated by the ROC curve analysis (Fig. 1).

Table 1 Hematological parameters of the patients

\begin{tabular}{lll}
\hline Hematological parameters & Range & Median \\
\hline Hemoglobin (gm\%) & 6.3 to 18 & 12.6 \\
Platelets $\left(\times 10^{9} / \mathrm{L}\right)$ & 30 to 600 & 210 \\
TLC $\left(\times 10^{9} / \mathrm{L}\right)$ & 2.6 to 30 & 11.2 \\
Neutrophil (\%) & 41 to 97 & 86 \\
Lymphocytes (\%) & 1 to 54 & 9.08 \\
NLR & 0.75 to 96 & 9.3 \\
Absolute eosinophil count (/cumm) & 0 to 812 & 43 \\
\hline
\end{tabular}


Fig. 1 ROC curve analysis of age, TLC, and NLR for predicting ICU admission

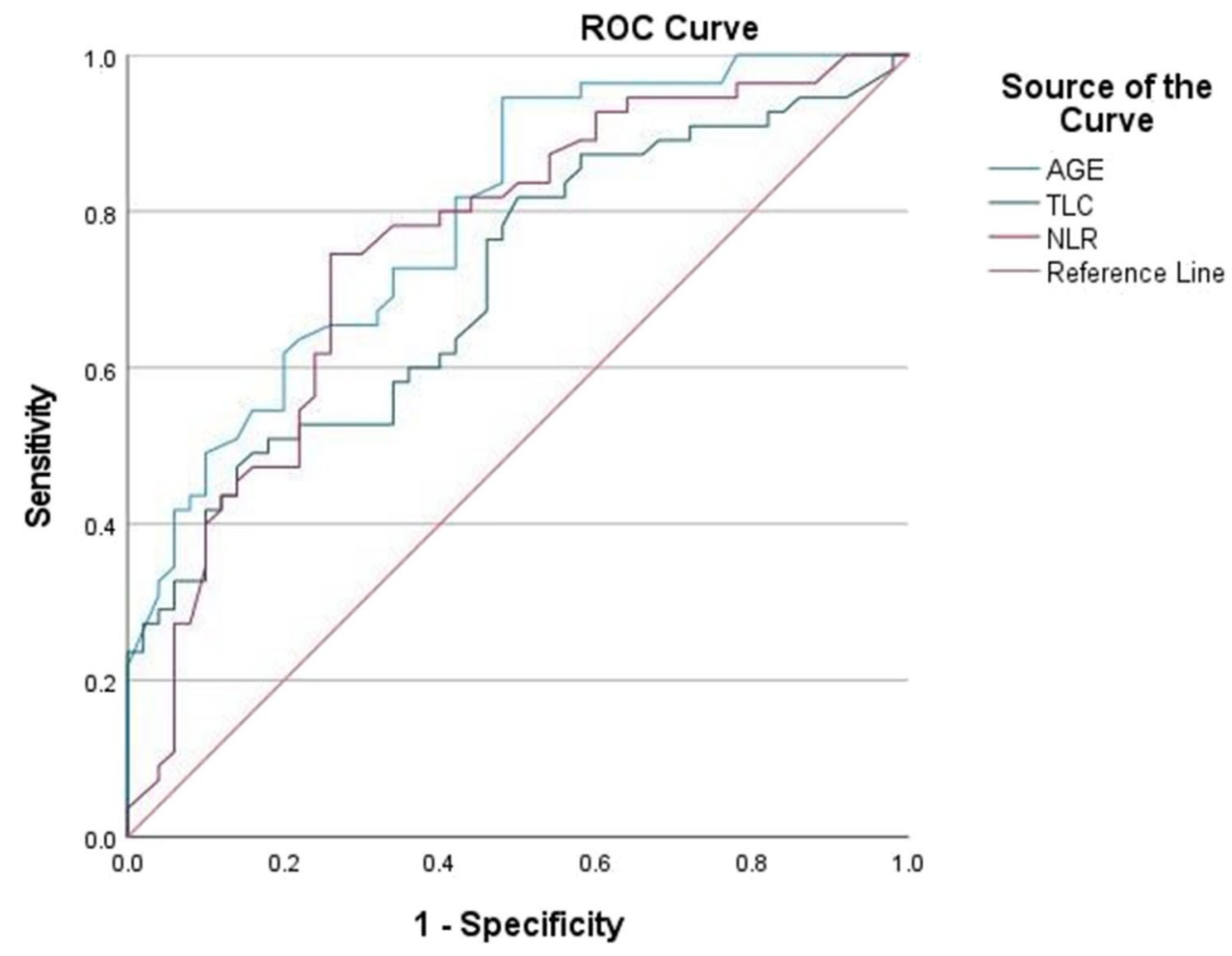

The area under the curve (AUC) of age, TLC, and NLR were $0.79,0.70$, and 0.75 respectively. The highest sensitivity for age was $72 \%$ and specificity was $66 \%$. The highest sensitivity and specificity for TLC were $60 \%$ and $64 \%$ respectively, and for NLR were $74 \%$ and $74 \%$ respectively (Table 2). With the highest sensitivity and specificity, the optimal cut-off values for age, TLC, and NLR were 47.5 years, $11.3 \times 10^{9} / \mathrm{L}$, and 9.1 respectively (Table 2 ).

All the parameters of the patients were analyzed with relation to ICU and non-ICU admissions (Table 3 ). Age $\geq 47.5(p<0.01)$, male patients $(p<0.001)$, TLC $\geq 11.3 \times 10^{9} / \mathrm{L} \quad(p<0.014)$, relative neutrophilia $\geq 70 \%(p<0.007)$, relative lymphopenia $\leq 20 \%$ $(p<0.002)$, NLR $\geq 9.1(p<0.001)$, and AEC $\leq 40$ /cumm $(p<0.001)$ were significantly associated with ICU admission (Table 3). AEC was zero in 35 out of 55 patients (63.6\%), who required ICU admission.

Hemoglobin levels $(p=0.29)$ and platelet counts $(p=0.61)$ had no correlation with regard to ICU admission of COVID-19 patients.

\section{Discussion}

In our study, we analyzed hemoglobin, platelet count, TLC, neutrophils, lymphocytes, NLR, and AEC to know their predictive role in the severity of COVID-19 patients. These hematological parameters within $24 \mathrm{~h}$ of admission were analyzed with relation to ICU and non-ICU admission of these patients.

In our study, optimal thresholds for age, TLC, and NLR were calculated by using the ROC curve. With the highest sensitivity and specificity, the optimal cut-off values for age, TLC, and NLR were calculated as 47.5 years, $11.3 \times 10^{9} / \mathrm{L}$, and 9.1 respectively. Those patients whose age $\geq 47.5$ years, TLC $\geq 11.3 \times 10^{9} / \mathrm{L}$, and NLR $\geq 9.1$ predicted the development of critical illness and required ICU admission for COVID-19 patients. A study done by Yang AP et al. showed that $46.1 \%$ of the COVID-19 patients with mild disease whose age $\geq 49.5$ years old and NLR $\geq 3.3$ became severe. So, these patients must be
Table 2 Area under the curve (AUC) for age, TLC, and NLR for predicting ICU admission

\begin{tabular}{lllllll}
\hline $\begin{array}{l}\text { Test result } \\
\text { variable(s) }\end{array}$ & AUC & $95 \%$ confidence interval & $p$-value & Optimal cut-off value & $\begin{array}{l}\text { Sensitiv- } \\
\text { ity }(\%)\end{array}$ & $\begin{array}{l}\text { Speci- } \\
\text { ficity } \\
(\%)\end{array}$ \\
\hline Age & 0.793 & $0.710-0.877$ & $<0.001$ & 47.5 years & 72 & 66 \\
TLC & 0.707 & $0.608-0.805$ & $<0.001$ & $11.3 \times 10^{9} / \mathrm{L}$ & 60 & 64 \\
NLR & 0.754 & $0.660-0.848$ & $<0.001$ & 9.1 & 74 & 74 \\
\hline
\end{tabular}


Table 3 Association of hematological parameters with COVID-19 patients

\begin{tabular}{lllll}
\hline Characteristics & & ICU $(n=55)$ & $\begin{array}{l}\text { Non- } \\
\text { ICU } \\
(n=50)\end{array}$ & $p$-value \\
& & & 17 & $<0.01$ \\
Age & $\geq 47.5$ years & 40 & 33 & \\
& $<47.5$ years & 15 & 16 & $<0.001$ \\
Sex & Male & 38 & 34 & \\
& Female & 17 & 34 & 0.29 \\
Hemoglobin & $\geq 12$ & 32 & 16 & \\
$($ gm\% $)$ & $<12$ & 23 & 46 & 0.61 \\
Platelets & $>150$ & 49 & 4 & \\
$\left(\times 10^{9} / \mathrm{L}\right)$ & $\leq 150$ & 6 & 18 & $<0.014$ \\
TLC & $\geq 11.3$ & 33 & 32 & \\
$\left(\times 10^{9} / \mathrm{L}\right)$ & $<11.3$ & 22 & 38 & $<0.007$ \\
$\begin{array}{l}\text { Neutrophil } \\
(\%)\end{array}$ & $\geq 70$ & 52 & 12 & \\
Lymphocytes & $>70$ & 3 & 18 & $<0.002$ \\
$(\%)$ & $\leq 20$ & 6 & 32 & \\
NLR & $\geq 9.1$ & 41 & 15 & $<0.001$ \\
& $<9.1$ & 14 & 35 & \\
Absolute eosino- & $>40$ & 17 & 35 & $<0.001$ \\
phil count $($ per & $\leq 40$ & 38 & 15 & \\
cumm) & & & & \\
\hline
\end{tabular}

closely attended to by clinicians [1]. In another series of 81 patients, Ma A et al. identified that NLR helped to predict the development of acute respiratory distress syndrome (ARDS) in COVID-19 patients [6]. They found that NLR $>11$ predicted the development of moderate to severe ARDS and NLR $>9.8$ could predict the overall requirement of ventilation [6]. In another prospective cohort of COVID-19 patients from Wuhan, China, by Liu J et al., a relatively lower NLR value of $>3.13$ predicted the development of critical illness in patients aged $>50$ years [7].

In the present study, relative neutrophilia $\geq 70 \%$ $(p<0.007)$, relative lymphopenia $\leq 20 \%(p<0.002)$, and NLR $\geq 9.1(p<0.001)$ were significantly associated with ICU admission. Neutrophils are a vital component of body immunity while lymphocytes play an important role in inflammatory responses. Despite their beneficial effect on the initiation of the adaptive response, neutrophils have been implicated in the development of ARDS via their role in the state of "hyper-cytokinemia" or cytokine storm in COVID-19 patients [8-10]. The production of cytotoxic T-lymphocytes (CTL) is a crucial part of the immune response during a viral infection [8]. Like its predecessors, SARS-CoV-2 infection is associated with a rapid fall in lymphocyte count, which is more pronounced in ICU patients [8, 11-13]. The different hypotheses explaining lymphopenia in COVID-19 are chemokine-mediated lymphocyte redistribution, sequestration in the lungs, and
CD13- or CD66-mediated bone marrow suppression [8, 11-13].

An elevated NLR suggests a dysregulation of the inflammatory and immune responses. Thus, an elevated NLR could be used as a probable marker of disease severity in infectious illnesses. In a meta-analysis of 15 studies by Zeng $\mathrm{F}$ et al., higher neutrophil counts and NLR but lower lymphocyte counts were observed in severe cases of COVID-19 compared to non-severe cases [14]. The significance of NLR in the prognosis of viral diseases like influenza patients was shown to be a more sensitive indicator compared to neutrophil or lymphocyte counts alone [15]. Yang AP et al. also reiterated NLR as an independent predictor of clinical outcomes in COVID-19 patients [1]. Similarly, Lagunas-Rangel FA et al. found a significant association of higher NLR in severe cases of COVID-19 [16]. These results corroborate the increasing clinical evidence on the predictive and prognostic value of NLR in COVID-19 patients.

Reduction of AEC was significantly associated with ICU admission in our study $(p<0.001)$. Eosinopenia is known to be associated with physiological stress response and acute inflammatory and systemic response [17, 18]. Therefore, it has been found in physiological stress and in several clinical conditions including sepsis, viral infections, corticosteroids and catecholamines therapy, psychiatric condition, and in some allergies $[19,20]$. It has been shown that the decrease of circulating eosinophils occurs rapidly after the initiation of an inflammatory process pointing to peripheral splenic sequestration and also sequestration at the site of infection due to the chemotactic effects of increased cytokines $[17,21]$. It has been observed that the indicators of classic inflammation, including c-reactive protein (CRP), ferritin, and multiple serum cytokines (IL-2R, IL-6, IL-8, IL-10, and TNF- $\alpha$ ), were significantly higher in the group having eosinopenia [22]. Jinjin $\mathrm{H}$ et al. demonstrated that AEC was zero in $61 \%$ of the patients who eventually required ICU care [22]. This event was called "the almost zero eosinophil effect," probably because of the process of "hyper-cytokinemia" caused by severe infection [23].

In the present study, AEC was zero in $63.6 \%$ who required ICU admission. Other studies also demonstrated that severe COVID-19 patients had much lower AEC and highly correlated with ICU transfer [22, 24]. Therefore, it is suggested that AEC could be a reasonable predictive marker for severity in COVID-19 patients.

The present study had a few limitations, which include selection bias due to the hospital-based population, a small sample size, a retrospective study design, and the lack of follow-up of the patients along with the serial assessment of the CBC parameters.

Routine hematological parameters are cost-effective, convenient, and fast predictive markers for severe COVID19 patients, which are helpful for resource-constrained 
health care facilities to utilize limited ICU resources more reasonably.

Author Contribution AS, BD, VR and, PD acquired and analyzed the data. BB, IT, PD, BSL, and KD conceptualized the manuscript. All the authors drafted, critically analyzed, and approved the final version of the manuscript.

Data availability Available.

Code Availability Available.

\section{Declarations}

Ethics Approval All procedures performed in studies involving human participants were in accordance with the ethical standards of the institutional and/or national research committee and with the 1964 Helsinki declaration and its later amendments or comparable ethical standards. The study was approved by the institute ethics committee under the project P208/2021/208 dated 24th August 2021.

Consent to Participate Not applicable.

Consent for Publication Not applicable.

Conflict of Interest The authors declare no competing interests.

\section{References}

1. Yang AP, Liu JP, Tao WQ, Li HM. The diagnostic and predictive role of NLR, d-NLR and PLR in COVID-19 patients. Int Immunopharmacol. 2020;84:106504.

2. Khourssaji M, Chapelle V, Evenepoel A, Belkhir L, Yombi JC, van Dievoet MA, et al. A biological profile for diagnosis and outcome of COVID-19 patients. Clin Chem Lab Med. 2020;58(12):2141-50.

3. Nair PR, Maitra S, Ray BR, Anand RK, Baidya DK, Subramaniam R. Neutrophil-to-lymphocyte ratio and platelet-to-lymphocyte ratio as predictors of the early requirement of mechanical ventilation in COVID-19 patients. Indian J Crit Care Med. 2020;24(11):1143-4.

4. Revised Guidelines on Clinical Management of COVID - 19. Available from: https://www.mohfw.gov.in. Accessed on 26th October 2021.

5. Saurabh A, Dey B, Raphael V, Deb P, Khonglah Y, Tiewsoh I. Role of coagulation profile in predicting disease severity among patients of COVID-19. Cureus. 2021;13(10):e19124.

6. Ma A, Cheng J, Yang J, Dong M, Liao X, Kang Y. Neutrophil-tolymphocyte ratio as a predictive biomarker for moderate-severe ARDS in severe COVID-19 patients. Crit Care. 2020;20(1):288.

7. Liu J, Liu Y, Xiang P, Pu L, Xiong H, Li C, et al. Neutrophil-tolymphocyte ratio predicts critical illness patients with 2019 coronavirus disease in the early stage. J Tranl Med. 2020;18(1):206.

8. He Z, Zhao C, Dong Q, Zhuang H, Song S, Peng G, et al. Effects of severe acute respiratory syndrome (SARS) coronavirus infection on peripheral blood lymphocytes and their subsets. Int J Infect Dis. 2005;9:323-30.
9. Abraham E. Neutrophils and acute lung injury. Crit Care Med. 2003;31(4 Suppl):S195-9.

10. Van den Brand JM, Haagmans BL, van Riel D, Osterhaus AD, Kuiken T. The pathology and pathogenesis of experimental severe acute respiratory syndrome and influenza in animal models. $\mathrm{J}$ Comp Pathol. 2014;151:83-112.

11 Wong RS, Wu A, To KF, Lee N, Lam CW, Wong CK, et al. Haematological manifestations in patients with severe acute respiratory syndrome: retrospective analysis. BMJ. 2003;326(7403):1358-62.

12. Panesar NS. What caused lymphopenia in SARS and how reliable is the lymphokine status in glucocorticoid-treated patients? Med Hypotheses. 2008;71(2):298-301.

13. Yang M, Li CK, Li K, Hon KL, Ng MH, Chan PK, et al. Hematological findings in SARS patients and possible mechanisms (review). Int J Mol Med. 2004;14(2):311-5.

14. Zeng F, Li L, Zeng J, Deng Y, Huang H, Chen B, et al. Can we predict the severity of COVID-19 with a routine blood test? Pol Arch Intern Med. 2020;130(5):400-6.

15. Ding X, Yu Y, Lu B, Huo J, Chen M, Kang Y, et al. Dynamic profile and clinical implications of hematological parameters in hospitalized patients with coronavirus disease 2019. Clin Chem Lab Med. 2020;58(8):1365-71.

16. Lagunas-Rangel FA. Neutrophil-to-lymphocyte ratio and lymphocyte-to-C-reactive protein ratio in patients with severe coronavirus disease 2019 (COVID-19): a meta-analysis. J Med Virol. 2020;92(10):1733-4.

17 Bass DA. Behavior of eosinophil leukocytes in acute inflammation. II. Eosinophil dynamics during acute inflammation. J Clin Invest. 1975;56(4):870-9.

18. Escobar-Valdivia EJ, González-Aguirre JE, Carrillo-Cisneros ER, Guerra-Leza KC, Mercado-Longoría R. Eosinophil count at intensive care unit admission was not predictor of hospital mortality: results of a case control study. J Intensive Care. 2015;3(1):27.

19. Zhang JJ, Dong X, Cao YY, Yuan Y, Yang Y, Yan Y, et al. Clinical characteristics of 140 patients infected with SARS-CoV-2 in Wuhan. China Allergy. 2020;75(7):1730-41.

20. Xu G, Yang Y, Du Y, Peng F, Hu P, Wang R, et al. Clinical pathway for early diagnosis of COVID-19: updates from experience to evidence-based practice. Clin Rev Allergy Immunol. 2020;59(1):89-100.

21. Bass DA, Gonwa TA, Szejda P, Cousart MS, DeChatelet LR, McCall CE. Eosinopenia of acute infection: production of eosinopenia by chemotactic factors of acute inflammation. J Clin Invest. 1980;65(6):1265-71.

22. Huang J, Zhang Z, Liu S, Gong C, Chen L, Ai G, et al. Absolute eosinophil count predicts intensive care unit transfer among elderly COVID-19 patients from general isolation wards. Front Med (Lausanne). 2020;7:585222.

23. Shaaban H, Daniel S, Sison R, Slim J, Perez G. Eosinopenia: is it a good marker of sepsis in comparison to procalcitonin and $\mathrm{C}$-reactive protein levels for patients admitted to a critical care unit in an urban hospital? J Crit Care. 2010;25(4):570-5.

24. Sun Y, Dong Y, Wang L, Xie H, Li B, Chang C, et al. Characteristics and prognostic factors of disease severity in patients with COVID-19: the Beijing experience. J Autoimmun. 2020; 112:102473.

Publisher's Note Springer Nature remains neutral with regard to jurisdictional claims in published maps and institutional affiliations. 